\title{
Síntese do Controle Linear Aplicado em Sistemas Populacionais ${ }^{1}$
}

A.B. SCHMID ${ }^{2}$, M. RAFIKOV ${ }^{3}$, Universidade Regional do Noroeste do Estado do Rio Grande do Sul -UNIJUÍ, Rua São Francisco 501, Cx.P. 560, 98700-000 Ijui, RS, Brasil.

Resumo. O objetivo deste trabalho é aplicar controle linear em sistemas populacionais que exibem caos para determinados valores dos parâmetros. São analisados dois sistemas: modelo de Lotka-Volterra para duas presas e um predador e modelo tritrófico de cadeia alimentar. A fim de encontrar o controle ótimo que estabiliza os sistemas nos pontos de equilíblio desejados foi aplicada a metodologia proposta por Rafikov e Balthazar. As simulações numéricas, que foram realizadas para os dois sistemas considerados, mostraram a possibilidade de controlá-los, utilizando controle linear.

\section{Introdução}

Durante várias décadas, depois do trabalho de Lotka [4] e Volterra [9], um dos tópicos de principal preocupação em ecologia matemática foi o estudo de cadeias alimentares ditróficas. Isto foi realizado analisando um grande número de modelos dinâmicos contínuos de segunda ordem, usualmente chamados modelos presapredador. Somente nos anos setenta que um pouco de interesse em modelos tritróficos de cadeia alimentar emergiu na matemática (composto de presa, predador, e super-predador).

Devido à relevância intrínseca para a biologia, estes modelos geraram, e ainda geram, uma quantia enorme de pesquisas, não só porque há muitos tipos de interações entre espécies para considerar, mas também porque até mesmo os modelos tritróficos mais simples possuem alguns problemas matemáticos desafiadores, acima de tudo, o caos é menos compreendido para estes modelos.

Nos últimos anos, o problema do controle de caos tornou-se de grande interesse. Entre as estratégias de controle de caos a mais popular é o método de OGY, no entanto, este usa o mapa de Poincaré e, por isso, tem limitações para sua aplicação.

Para estabilizar sistemas caóticos foi utilizado, em vários trabalhos, o controle linear feedback (Vicent,Yu [8], Bewley [2], Sinha et al. [6]). Mas, nestes, o uso do controle linear para sistemas não-lineares não foi bem justificado. Uma metodologia para encontrar o controle ótimo linear feedback foi proposta por Rafikov e Balthazar

\footnotetext{
${ }^{1}$ Trabalho de dissertação do curso de Mestrado em Modelagem Matemática.

2abs@detec.unijui.tche.br,; bolsista CAPES.

${ }^{3}$ rafikov@admijui.unijui.tche.br
} 
[5]. Eles encontraram as condições que garantem a aplicação do controle linear em sistemas não-lineares. Temos que notar que na teoria do controle existem dois tipos de problemas: o controle de programa e a síntese do controle. Para o primeiro, a função do controle $u(t)$ é encontrada como uma função do tempo. Ou seja, nesse caso a função de controle ótimo determina uma trajetória ótima que corresponde a uma condição inicial dada do sistema. No segundo caso, a função do controle $u(t, x)$ depende do tempo e de variáveis de estado. Este tipo de controle é chamado controle feedback ou controle com realimentação e pode ser aplicado para qualquer condição inicial. Se as variáveis do sistema são desvios do regime desejado, o controle ótimo estabiliza o sistema em torno da trajetória desejada, minimizando o funcional que caracteriza os desvios quadrados da trajetória e do controle do regime desejado.

Em vários problemas do controle de sistemas populacionais os regimes desejados dos sistemas são conhecidos. Por exemplo, no controle de pragas é conhecido o valor limiar de danos econômicos que determina a quantidade de pragas que não causam

danos econômicos na lavoura. As quantidades de inimigos naturais que sustentam as pragas neste limiar podem ser fornecidas por especialistas do controle biológico.

Em nosso trabalho foi formulado e resolvido o problema da síntese do controle para dois sistemas populacionais: Lotka-Volterra de duas presas e um predador e sistema tritrófico de cadeia alimentar.

\section{Formulação do Problema}

Consideramos um sistema controlado

$$
\dot{x}=A x+g(x)+U,
$$

onde $x \in R^{n}$ é um vetor de estado, $A \in R^{n x n}$ é uma matriz constante e $g(x)$ um vetor formado de funções contínuas, e o vetor de controle é

$$
U=\tilde{u}+u_{t} .
$$

Sem controle $(U \equiv 0)$ o sistema (2.1) apresenta comportamento caótico para determinados valores dos parâmetros. É de nosso interesse controlar o sistema, levando-o para um ponto ou trajetória desejada. Seja $\tilde{x}$ esta trajetória, então a parte $\tilde{u}$ do vetor de controle que mantém o sistema controlado na trajetória desejada, pode ser escrito como

$$
\tilde{u}=\dot{\tilde{x}}-A \tilde{x}-g(\tilde{x})
$$

e o vetor de controle $u_{t}$ que estabiliza o sistema em torno da órbita desejada tem a forma seguinte

$$
u_{t}=B u,
$$

onde $B \in R^{n x m}$ é uma matriz constante.

Definindo

$$
y=x-\tilde{x}
$$

como o desvio da trajetória do sistema (2.1) da trajetória desejada, e admitindo (2.2)-(2.4), chegamos à equação em desvios:

$$
\dot{y}=A y+g(x)-g(\tilde{x})+B u .
$$


A parte não-linear do sistema (2.5) pode ser escrita como

$$
g(x)-g(\tilde{x})=G(x, \tilde{x})(x-\tilde{x})
$$

onde $G(x, \tilde{x})$ é uma matriz limitada, cujos elementos dependem de $x$ e $\tilde{x}$. Admitindo (2.6), o sistema (2.5) tem a seguinte forma:

$$
\dot{y}=A y+G(x, \tilde{x})+B u .
$$

No trabalho [5] foi formulado o seguinte teorema: Se existem as matrizes $Q$ e $R$, definidas positivas, sendo $Q$ simétrica, tais que a matriz

$$
\tilde{Q}=Q-G^{T}(x, \tilde{x}) P-P G(x, \tilde{x})
$$

seja definida positiva para $G$ limitada, então o controle linear feedback

$$
u=-R^{-1} B^{T} P y
$$

é ótimo para transferir o sistema não linear (2.7)de qualquer estado inicial ao estado final

$$
y(\infty)=0,
$$

minimizando o funcional

$$
\tilde{J}=\int_{0}^{\infty}\left(y^{T} \tilde{Q} y+u^{T} R u\right) d t,
$$

onde a matriz simétrica $P$ é calculada da equação algébrica não linear de Riccati:

$$
P A+A^{T} P-P B R^{-1} B^{T} P+Q=0,
$$

onde as matrizes $Q \in R^{n x n}$ e $R \in R^{n x m}$ são constantes, definidas positivas.

É importante destacar que a matriz A na equação (2.5) não é única, e pode influenciar no desempenho do controlador.

Para resolver um problema de síntese de controle (2.7)-(2.11) pode ser utilizado o seguinte algoritmo colocado no problema acima referido:

1. Para as matrizes dadas $A$ e $Q$ resolver a equação algébrica de Riccati (2.12), encontrando a matriz $P$.

2. Calcular a função de controle (2.9).

3. Calcular as trajetórias ótimas, integrando o sistema (2.7).

4. Verificar se a condição (2.8) é satisfeita. Caso (2.8)não seja satisfeita, escolher outra matriz $Q$ e repetir os cálculos a partir do item 1 . 


\section{Controle Linear no Modelo de Lotka-Volterra de duas Presas e um Predador}

O modelo de Lotka-Volterra para duas presas e um predador é dado por:

$$
\frac{d x_{i}}{d t}=x_{i}\left(r_{i}-\sum c_{i j} x_{j}\right), \quad i, j=1,2,3,
$$

ou seja,

$$
\begin{gathered}
\dot{x_{1}}=x_{1}\left(r_{1}-c_{11} x_{1}-c_{12} x_{2}-c_{13} x_{3}\right) \\
\dot{x_{2}}=x_{2}\left(r_{2}-c_{21} x_{1}-c_{22} x_{2}-c_{23} x_{3}\right) \\
\dot{x_{3}}=x_{3}\left(r_{3}-c_{31} x_{1}-c_{32} x_{2}-c_{33} x_{3}\right),
\end{gathered}
$$

onde:

$x_{1}, x_{2}, x_{3}$ são as densidades da primeira presa, segunda presa e predador, respectivamente;

$c_{i j}, i, j=1, \ldots, n$ são coeficientes que admitem a interação entre as espécies da população;

$r_{i}$ é a taxa de crescimento da população $i$.

De acordo com Vance [7] e Gilpin [3] o sistema (3.1) apresenta comportamento caótico para os seguintes parâmetros: $r_{1}=r_{2}=-r_{3}=1, c_{11}=c_{12}=c_{22}=c_{23}=$ 0,$001 ; c_{21}=0,0015 ; c_{13}=0,01 ; c_{31}=-0,005 ; c_{32}=-0,0005 ; c_{33}=0$.

Introduzindo controle, temos

$$
\frac{d x_{i}}{d t}=x_{i}\left(r_{i}-\sum c_{i j} x_{j}\right)+\tilde{u}_{i}+u_{i}, \quad i, j=1,2,3,
$$

onde o controle feedforward $\tilde{u}$, conforme (2.3), tem a seguinte forma

$$
\tilde{u}_{i}=-\tilde{x}_{i}\left(r_{i}+\sum\left(c_{i j} \tilde{x}_{j}\right) .\right.
$$

O sistema Lotka-Volterra com desvios tem a forma

$$
\dot{y}=A y+G(x, \tilde{x}) y+B u,
$$

onde $A=\left(\begin{array}{ccc}r_{1} & 0 & 0 \\ 0 & r_{2} & 0 \\ 0 & 0 & r_{3}\end{array}\right), \quad B=\left(\begin{array}{ccc}1 & 0 & 0 \\ 0 & 1 & 0 \\ 0 & 0 & 1\end{array}\right)$ e os elementos de $G(x, \tilde{x})$ são:

$$
\begin{aligned}
& g_{11}=-c_{11}\left(x_{1}+\tilde{x_{1}}\right)-c_{12} x_{2}-c_{13} x_{3}, \quad g_{12}=-c_{12} \tilde{x}_{1}, \\
& g_{13}=-c_{13} \tilde{x}_{1}, \quad g_{21}=-c_{21} x_{2}, \\
& g_{22}=-c_{22}\left(x_{2}+\tilde{x_{2}}\right)-c_{21} \tilde{x}_{1}-c_{23} x_{3}, \quad g_{23}=-c_{23} \tilde{x}_{2}, \\
& g_{31}=-c_{31} x_{3}, \quad g_{32}=-c_{32} x_{3}, \\
& g_{33}=-c_{33}\left(x_{3}+\tilde{x_{3}}\right)-c_{31} \tilde{x}_{1}-c_{32} \tilde{x}_{2} .
\end{aligned}
$$

Escolhendo $Q=\left(\begin{array}{ccc}10 & 0 & 0 \\ 0 & 10 & 0 \\ 0 & 0 & 10\end{array}\right) \quad$ e $\quad R=\left(\begin{array}{ccc}1 & 0 & 0 \\ 0 & 1 & 0 \\ 0 & 0 & 1\end{array}\right)$, 
obtemos $P=\left(\begin{array}{ccc}4,3166 & 0 & 0 \\ 0 & 4,3166 & 0 \\ 0 & 0 & 2,3166\end{array}\right)$

resolvendo a equação algébrica de Riccati (2.12) através da função LQR do software MATLAB.

Assim, a função de controle ótimo para este sistema tem a seguinte forma:

$$
u=-\left(\begin{array}{ccc}
4,3166 & 0 & 0 \\
0 & 4,3166 & 0 \\
0 & 0 & 2,3166
\end{array}\right) y
$$

onde $y=\left(\begin{array}{c}x_{1}-\tilde{x_{1}} \\ x_{2}-\tilde{x_{2}} \\ x_{3}-\tilde{x_{3}}\end{array}\right)$.

As trajetórias do sistema controlado (3.2) com controle linear (3.4) estão na Figura 1(a). As simulações foram realizadas com condições iniciais $(10,20,1)$ e o ponto de equilíbrio escolhido foi $(8,10,4)$.

Atribuindo os valores máximos a $x_{1}, x_{2}$ e $x_{3}$ podemos avaliar a condição (2.8)

$$
\tilde{q}_{i i} \geq q_{i i}-2 g_{i i}^{\max } p_{i i}, \quad i=1,2,3,
$$

onde $g_{i i}^{\max }$ são calculados de (3.3) com os valores máximos de $x_{i}$. Como pode ser visto da Figura 1 os valores iniciais de $x_{1}$ e $x_{2}$ são máximos assim como o valor final de $x_{3}$. Então,

$$
\tilde{Q} \geq\left(\begin{array}{ccc}
10,67 & 0 & 0 \\
0 & 10,4 & 0 \\
0 & 0 & 9,79
\end{array}\right)>0 .
$$

Como $\tilde{Q}$ é definida positiva conclui-se baseado no teorema acima exposto que o controle (3.4) é ótimo.

Como já foi mencionado acima, a matriz $A$ não é única. A escolha de outra matriz pode influenciar na dinâmica do sistema controlado. Por exemplo, de acordo com [1], na estabilização de sistemas não-lineares, orienta-se usar a matriz $A$ do modelo linearizado, neste caso, dada por:

$$
\begin{array}{ll}
a_{11}=r_{1}-2 c_{11} \tilde{x}_{1}-c_{12} \tilde{x}_{2}-c_{13} \tilde{x}_{3}, & a_{12}=-\tilde{x}_{1} c_{12}, \\
a_{13}=-\tilde{x}_{1} c_{13}, & a_{21}=-\tilde{x}_{2} c_{21}, \\
a_{22}=r_{2}-c_{21} \tilde{x}_{1}-2 c_{22} \tilde{x}_{2}-c_{23} \tilde{x}_{3}, & a_{23}=-\tilde{x}_{2} c_{23}, \\
a_{31}=-\tilde{x}_{3} c_{31}, & a_{32}=-\tilde{x}_{3} c_{32}, \\
a_{33}=r_{3}-c_{31} \tilde{x}_{1}-c_{32} \tilde{x}_{2}-2 c_{33} \tilde{x}_{3}, &
\end{array}
$$

ou seja,

$$
A=\left(\begin{array}{ccc}
0,934 & -0,008 & -0,08 \\
-0,015 & 0,964 & -0,01 \\
0,02 & 0,002 & -0,955
\end{array}\right) \text {. }
$$


[a]

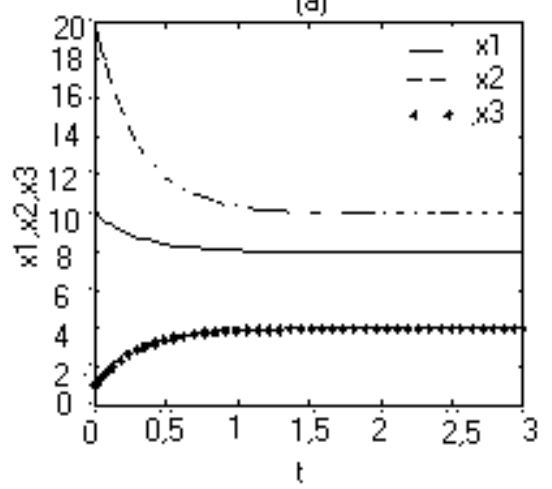

(b)

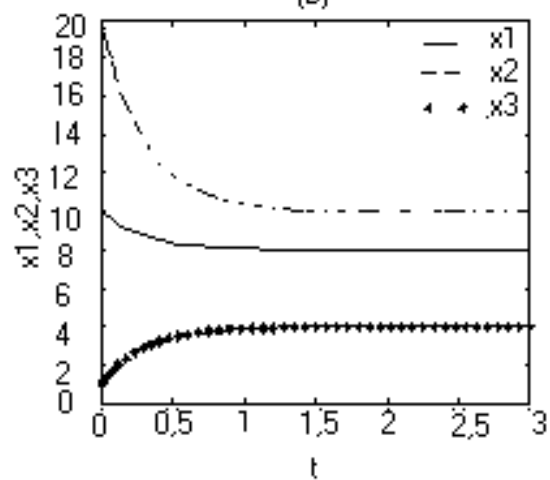

Figura 1: (a)Trajetórias do sistema Lotka-Volterra com controle linear (3.4). (b) Trajetórias do sistema Lotka-Volterra com controle linear (3.6).

Assim, temos

$$
\begin{array}{ll}
g_{11}=-c_{11}\left(x_{1}+\tilde{x_{1}}\right)-c_{12}\left(x_{2}-\tilde{x}_{2}\right)-c_{13}\left(x_{3}-\tilde{x}_{3}\right), & g_{12}=0, \\
g_{13}=0, & g_{21}=-c_{21}\left(x_{2}-\tilde{x}_{2}\right), \\
g_{22}=-c_{22}\left(x_{2}+\tilde{x_{2}}\right)+2 c_{22} \tilde{x}_{2}, & g_{23}=0, \\
g_{31}=-c_{31}\left(x_{3}-\tilde{x}_{3}\right), & g_{32}=-c_{32}\left(x_{3}-\tilde{x}_{3}\right), \\
g_{33}=-c_{33}\left(x_{3}+\tilde{x_{3}}\right)+2 c_{33} \tilde{x}_{3} . &
\end{array}
$$

Novamente, usando a função LQR do software MATLAB, obtemos

$$
P=\left(\begin{array}{ccc}
4,2308 & -0,0149 & -0,0441 \\
-0,0149 & 4,2699 & -0,0056 \\
-0,0441 & -0,0056 & 2,3491
\end{array}\right)
$$

A função de controle ótimo é

$$
\begin{aligned}
& u_{1}=-4,2308\left(x_{1}-8\right)+0,0149\left(x_{2}-10\right)+0,0441\left(x_{3}-4\right), \\
& u_{2}=0,0149\left(x_{1}-8\right)-4,2699\left(x_{2}-10\right)+0,0056\left(x_{3}-4\right), \\
& u_{3}=0,0441\left(x_{1}-8\right)+0,0056\left(x_{2}-10\right)-2,3491\left(x_{3}-4\right) .
\end{aligned}
$$

Calculando $\tilde{Q}$ utilizando agora (3.5), temos

$$
\tilde{Q} \geq\left(\begin{array}{ccc}
10,24 & 0 & 0 \\
-0,0045 & 10,08 & 0 \\
0 & 0 & 10
\end{array}\right)>0
$$

As trajetórias do sistema controlado (3.2) com a função de controle (3.6), ou seja, utilizando a matriz A linearizada estão na Figura 1(b).

A comparação dos gráficos na Figura 1 mostra que a alteração da matriz $A$, neste caso, quase não influi na dinâmica do sistema controlado. 


\section{Controle Linear Feedback para o Modelo Tritró- fico de Cadeia Alimentar}

O modelo de cadeia alimentar para três espécies com controle é dado por:

$$
\begin{aligned}
& \dot{x_{1}}=x_{1}\left(r-\frac{r x_{1}}{k}-\frac{a_{1} x_{2}}{1+b_{1} x_{1}}\right)+\tilde{u_{1}}+u_{1} \\
& \dot{x_{2}}=x_{2}\left(\frac{a_{1} x_{1}}{1+b_{1} x_{1}}-d_{2}-\frac{a_{2} x_{3}}{1+b_{2} x_{2}}\right)+\tilde{u_{2}}+u_{2}, \\
& \dot{x_{3}}=x_{3}\left(\frac{a_{2} x_{2}}{1+b_{2} x_{2}}-d_{3}\right)+\tilde{u_{3}}+u_{3}
\end{aligned}
$$

onde:

$r$ é a taxa de crescimento intrínseco; $k$ é a capacidade suporte das espécies;

$a_{1}$ e $a_{2}$ representam a taxa máxima de predação;

$d_{2}$ e $d_{3}$ são taxas de mortalidade;

$b_{1}$ e $b_{2}$ são taxas de saturação.

Podemos escrever o sistema tritrófico de cadeia alimentar na forma de desvios como

$$
\dot{y}=A y+G(x, \tilde{x}) y+B u
$$

onde

$$
\begin{aligned}
& A=\left(\begin{array}{ccc}
r & 0 & 0 \\
0 & -d_{2} & 0 \\
0 & 0 & -d_{3}
\end{array}\right), \quad B=\left(\begin{array}{ccc}
1 & 0 & 0 \\
0 & 1 & 0 \\
0 & 0 & 1
\end{array}\right) \text { e os elementos de } G(x, \tilde{x}) \text { são } \\
& g_{11}=-\frac{r}{k}\left(x_{1}+\tilde{x_{1}}\right)-\frac{a_{1} x_{2}}{\left(1+b_{1} x_{1}\right)\left(1+b_{1} \tilde{x_{1}}\right)}, \quad g_{12}=\frac{-a_{1} b_{1} x_{1} \tilde{x_{1}}-a_{1} \tilde{x_{1}}}{\left(1+b_{1} x_{1}\right)\left(1+b_{1} \tilde{x_{1}}\right)}, \\
& g_{13}=0 \text {, } \\
& g_{21}=\frac{a_{1} x_{2}}{\left(1+b_{1} x_{1}\right)\left(1+b_{1} \tilde{x_{1}}\right)}, \\
& g_{22}=\frac{a_{1} b_{1} x_{1} \tilde{x_{1}}-a_{1} \tilde{x_{1}}}{\left(1+b_{1} x_{1}\right)\left(1+b_{1} \tilde{x_{1}}\right)}-\frac{a_{2} x_{3}}{\left(1+b_{2} x_{2}\right)\left(1+b_{2} \tilde{x_{2}}\right)}, \quad g_{23}=\frac{-a_{2} b_{2} x_{2} \tilde{x_{2}}+a_{2} \tilde{x_{2}}}{\left(1+b_{2} x_{2}\right)\left(1+b_{2} \tilde{x_{2}}\right)}, \\
& g_{31}=0 \text {, } \\
& g_{32}=\frac{a_{2} x_{3}}{\left(1+b_{2} x_{2}\right)\left(1+b_{2} \tilde{x_{2}}\right)} \text {, } \\
& g_{33}=\frac{a_{2} b_{2} x_{2} \tilde{x_{2}}+a_{2} \tilde{x_{2}}}{\left(1+b_{2} x_{2}\right)\left(1+b_{2} \tilde{x_{2}}\right)} .
\end{aligned}
$$

Os parâmetros usados nas simulações foram os seguintes $a 1=5 ; a 2=0.1, b 1=$ $3.2 ; b 2=2, d 2=0.45, d 3=0.01, k=10, r=0.9022$.

$$
\begin{aligned}
\text { Escolhendo } \quad Q= & \left(\begin{array}{ccc}
10 & 0 & 0 \\
0 & 10 & 0 \\
0 & 0 & 10
\end{array}\right) \quad \text { e } \\
P & =\left(\begin{array}{ccc}
4,1907 & 0 & 0 \\
0 & 2,7441 & 0 \\
0 & 0 & 3,1523
\end{array}\right),\left(\begin{array}{lll}
1 & 0 & 0 \\
0 & 1 & 0 \\
0 & 0 & 1
\end{array}\right) \text {, obtemos }
\end{aligned}
$$

resolvendo a equação algébrica de Riccati(2.12) através da função LQR do software MATLAB.

Assim, a função de controle ótimo para este sistema tem a seguinte forma:

$$
u=-\left(\begin{array}{ccc}
4,1907 & 0 & 0 \\
0 & 2,7441 & 0 \\
0 & 0 & 3,1523
\end{array}\right) y
$$


onde $y=\left(\begin{array}{c}x_{1}-\tilde{x_{1}} \\ x_{2}-\tilde{x_{2}} \\ x_{3}-\tilde{x_{3}}\end{array}\right)$.

As trajetórias do sistema controlado (4.2) com controle linear (4.4) estão na Figura 2 (a). Condições iniciais $(10,20,1)$ e ponto de equilíbrio $(8,10,4)$.

Atribuindo os valores máximos a $x_{1}, x_{2}$ e $x_{3}$ podemos avaliar a condição (2.8). Então,

$$
\tilde{Q} \geq\left(\begin{array}{ccc}
24,50 & 0 & 0 \\
0 & 2,27 & 0 \\
0 & 0 & 9,685
\end{array}\right)>0 .
$$

Como $Q$ é definida positiva conclui-se baseado no teorema acima exposto que o controle (4.4) é ótimo.

Para o modelo linearizado a matriz A é dada por:

$$
A=\left(\begin{array}{ccc}
r-\frac{2 r \tilde{x}_{1}}{k}-\frac{a_{1} \tilde{x}_{2}}{\left(1+b_{1} \tilde{x}_{1}\right)^{2}} & -\frac{\tilde{x}_{1} a_{1}}{1+b_{1} \tilde{x}_{1}} & 0 \\
\frac{a_{1} \tilde{x}_{2}}{\left(1+b_{1} \tilde{x}_{1}\right)^{2}} & \frac{\tilde{x}_{1} a_{1}}{1+b_{1} \tilde{x}_{1}}-d_{2}-\frac{\tilde{x}_{3} a_{2}}{1+b_{2} \tilde{x}_{2}} & -\frac{\tilde{x}_{2} a_{2}}{1+b_{2} \tilde{x}_{2}} \\
0 & \frac{\tilde{x}_{3} a_{2}}{\left(1+b_{2} \tilde{x}_{2}\right)^{2}} & \frac{\tilde{x}_{2} a_{2}}{1+b_{2} \tilde{x}_{2}}-d_{3}
\end{array}\right)
$$

ou seja,

$$
A=\left(\begin{array}{ccc}
-0,61 & -1,5 & 0 \\
0,07 & 1,05 & -0,05 \\
0 & 0,001 & 0,038
\end{array}\right)
$$

Assim, temos

$$
\begin{aligned}
& g_{11}=-\frac{r}{k}\left(x_{1}+\tilde{x_{1}}\right)-\frac{a_{1} x_{2}}{\left(1+b_{1} x_{1}\right)\left(1+b_{1} \tilde{x_{1}}\right)}+\frac{2 r \tilde{x}_{1}}{k}+\frac{a_{1} \tilde{x}_{2}}{\left(1+b_{1} \tilde{x_{1}}\right)^{2}}, \quad g_{12}=0 \\
& g_{13}=0, \quad g_{21}=\frac{a_{1} x_{2}}{\left(1+b_{1} x_{1}\right)\left(1+b_{1} \tilde{x_{1}}\right)}-\frac{a_{1} \tilde{x}_{2}}{\left(1+b_{1} \tilde{x_{1}}\right)^{2}}, \\
& g_{22}=\frac{-2 a_{1} \tilde{1}}{\left(1+b_{1} x_{1}\right)\left(1+b_{1} \tilde{x_{1}}\right)}-\frac{a_{2} x_{3}}{\left(1+b_{2} x_{2}\right)\left(1+b_{2} \tilde{x_{2}}\right)}+\frac{a_{2}}{\left(1+b_{2} \tilde{x_{2}}\right)^{2}}, \quad g_{23}=\frac{2 a_{2} \tilde{x_{2}}}{\left(1+b_{2} x_{2}\right)\left(1+b_{2} \tilde{x_{2}}\right)}, \\
& g_{31}=0, \quad g_{32}=\frac{a_{2} x_{3}}{\left(1+b_{2} x_{2}\right)\left(1+b_{2} \tilde{x_{2}}\right)}-\frac{a_{2} \tilde{x}_{3}}{\left(1+b_{2} \tilde{x_{2}}\right)^{2}}, \\
& g_{33}=0 .
\end{aligned}
$$

Novamente, usando a função LQR do software MATLAB, obtemos

$$
P=\left(\begin{array}{ccc}
2,5558 & -0,5254 & 0,001 \\
-0,5254 & 4,5716 & -0,0339 \\
0,001 & -0,0339 & 3,2009
\end{array}\right)
$$

A função de controle ótimo é, então,

$$
\begin{aligned}
& u_{1}=-2,5558\left(x_{1}-8\right)+0,5254\left(x_{2}-10\right)-0,001\left(x_{3}-4\right) \\
& u_{2}=0,5254\left(x_{1}-8\right)-4,5716\left(x_{2}-10\right)+0,0339\left(x_{3}-4\right) \\
& u_{3}=-0,001\left(x_{1}-8\right)+0,0339\left(x_{2}-10\right)-3,2009\left(x_{3}-4\right)
\end{aligned} .
$$

Calculando $\tilde{Q}$ utilizando agora (4.5), temos

$$
\tilde{Q} \geq\left(\begin{array}{ccc}
11,14 & 0 & 0 \\
0,045 & 10,82 & 0,00014 \\
0 & -0,00003 & 10
\end{array}\right)>0
$$


[a]

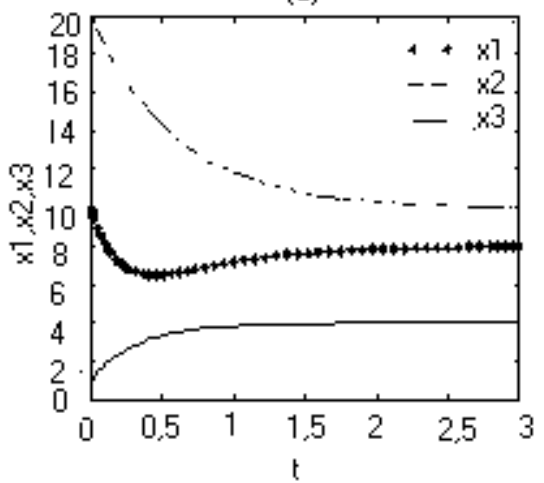

(b)

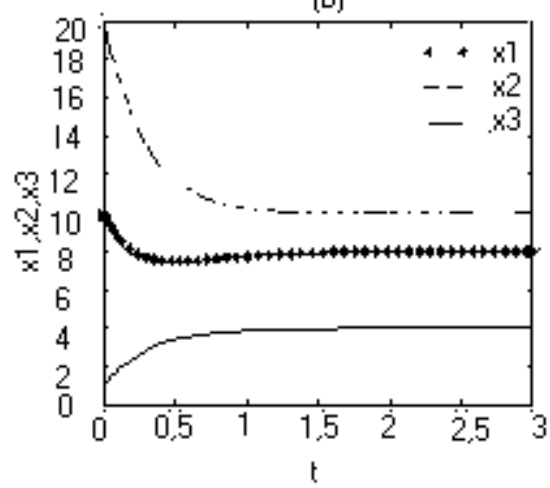

Figura 2: (a)Trajetórias do sistema de cadeia alimentar com controle linear (4.4). (b) Trajetórias do sistema de cadeia alimentar com controle linear(4.6).

A comparação dos gráficos na Figura 2 mostra que a alteração da matriz $A$, neste caso, influi na dinâmica do sistema controlado.

\section{Conclusões}

Foram analisados dois sistemas: modelo de Lotka-Volterra de duas presas e um predador e modelo de cadeia alimentar, ambos para três espécies que exibem caos para determinados valores dos parâmetros.

A metodologia proposta neste trabalho permitiu resolver o problema do controle de populações para os dois modelos considerados. As simulações computacionais mostraram que os algoritmos, propostos de aplicação do controle linear para modelos não-lineares, permitem, variando os valores de elementos da matriz $\mathrm{Q}$, melhorar a qualidade de trajetórias de transição e estabilizar os sistemas não-lineares no ponto de equilíbrio desejado.

Apesar de serem escolhidos dois sistemas que exibem caos, o controle ótimo proposto pode ser aplicado para sistemas não-lineares que não exibem caos.

Comparando as Figuras 1(a) e (b) observa-se que no modelo de Lotka-Volterra não há praticamente nenhuma diferença alterando a matriz $A$. No entanto, esta diferença existe para o modelo de cadeia alimentar como pode ser observado nas Figuras 2(a) e (b).

\section{Agradecimentos}

À CAPES pelo apoio financeiro cedido para a realização da pesquisa de mestrado.

\footnotetext{
Abstract.The objective of this work is to apply linear optimal control in population systems that exhibit chaos for certain values of the parameters. Two systems are analyzed: the two preys and one predator Lotka-Volterra model and the tritrophic food chain model. In order to find the optimal control that stabilizes the systems in the desired equilibrium points, the methodology, proposed by Rafikov and Balthazar, was applied. The numeric simulations, that were accomplished for the two considered systems, showed the possibility to control them, using linear control.
} 


\section{Referências}

[1] E.A. Barbashin, "Lyapunov Functions", Nauka, Moscow, 1970 (in Russian).

[2] T.R. Bewley, Linear control and estimation of nonlinear chaotic convection: Harnessing the batterfly effect, Physics of fluids., 11, No. 5 (1991), 896-903.

[3] M.E. Gilpin, Spiral chaos in a predador - prey model, American Naturalist, 113 (1979), 306-308.

[4] A. Lotka, "Elements of Physical Biology", Williams and Wilkins, Baltimore, MD, 1925.

[5] M. Rafikov e J.M.Balthazar, Síntese do controle ótimo linear feedback para sistemas que exibem caos. em "Anais do III Congresso Temático de Dinâmica, Controle e Aplicações", pp. 619-633, Ilha Solteira, SP, 2004.

[6] S.C. Sinha, J.T. Henrichs e B. Ravindra, A geral approach in the disign of active controllers for nonlinear systems exhiting chaos, International Journal of Bifurcation and Chaos, 10, No. 1 (2000), 165-178.

[7] R.R. Vance, Predation and resource partitioning in one predador - two prey, model community, American Naturalist, (1978), 797-813.

[8] T.L. Vincent; J. Yu, Control of a chaotic system, Dyn. Control, 1 (1991), 35-52.

[9] V. Volterra, Variazioni e uttuazioni del numero di individui in specie animali conviventi, Mem. Accad. Lincei, 2 (1926), 31-113. (In Italian). 